\title{
Patient-Specific Computational Model and Dosimetry Calculations for PET/CT of a Patient Pregnant with Twins
}

\author{
Tianwu Xie ${ }^{1}$, Paolo Zanotti-Fregonara ${ }^{2}$, Agathe Edet-Sanson ${ }^{3}$, and Habib Zaidi ${ }^{1,4-6}$ \\ ${ }^{1}$ Division of Nuclear Medicine and Molecular Imaging, Geneva University Hospital, Geneva, Switzerland; ${ }^{2}$ Houston Methodist \\ Research Institute, Houston, Texas; ${ }^{3}$ Department of Nuclear Medicine, Centre Henri Becquerel, Rouen, France; ${ }^{4}$ Department of \\ Nuclear Medicine and Molecular Imaging, University Medical Center Groningen, Groningen, Netherlands; ${ }^{5}$ Geneva University \\ Neurocenter, University of Geneva, Geneva, Switzerland; and ${ }^{6}$ Department of Nuclear Medicine, University of Southern Denmark, \\ Odense, Denmark
}

\begin{abstract}
The radiation dose delivered to pregnant patients during radiologic imaging procedures raises health concerns because the developing embryo and fetus are considered to be highly radiosensitive. To appropriately weigh the diagnostic benefits against the radiation risks, the radiologist needs reasonably accurate and detailed estimates of the fetal dose. Expanding our previously developed series of computational phantoms for pregnant women, we here describe a personalized model for twin pregnancy, based on an actual clinical scan. Methods: The model is based on a standardized hybrid pregnant female and fetus phantom and on a clinical case of a patient who underwent an ${ }^{18} \mathrm{~F}-\mathrm{FDG}$ PET/CT scan while expecting twins at 25 weeks' gestation. This model enabled us to produce a realistic physical representation of the pregnant patient and to estimate the maternal and fetal organ doses from the ${ }^{18} \mathrm{~F}$ FDG and CT components. The Monte Carlo N-Particle Extended general-purpose code was used for radiation transport simulation. Results: The ${ }^{18} \mathrm{~F}-\mathrm{FDG}$ doses for the 2 fetuses were 3.78 and 3.99 mGy, and the CT doses were 0.76 and 0.70 mGy, respectively. Therefore, the relative contribution of ${ }^{18} \mathrm{~F}-\mathrm{FDG}$ and CT to the total dose to the fetuses was about $84 \%$ and $16 \%$, respectively. Meanwhile, for ${ }^{18} \mathrm{~F}-\mathrm{FDG}$, the calculated personalized absorbed dose was about $40 \%-50 \%$ higher than the doses reported by other dosimetry computer software tools. Conclusion: Our approach to constructing personalized computational models allows estimation of a patient-specific radiation dose, even in cases with unusual anatomic features such as a twin pregnancy. Our results also show that, even in twins, the fetal organ doses from both ${ }^{18} \mathrm{~F}-\mathrm{FDG}$ and CT present a certain variability linked to the anatomic characteristics. The CT fetal dose is smaller than the ${ }^{18} \mathrm{~F}-\mathrm{FDG}$ PET dose.
\end{abstract}

Key Words: CT; radiation dosimetry; fetus; pregnant female models; Monte Carlo simulation

J Nucl Med 2018; 59:1451-1458

DOI: 10.2967/jnumed.117.205286

\section{$\mathbf{I}$} pregnant patients, it is critical that the benefit from radiologic examinations be carefully weighed against the possible radiation risk to the fetus. At fetal doses greater than 50-100 mGy, which

Received Nov. 13, 2017; revision accepted Jan. 12, 2018.

For correspondence or reprints contact: Habib Zaidi, Geneva University Hospital, 24 rue Micheli-du-Crest, $\mathrm{CH}-1211$ Geneva, Switzerland.

E-mail: habib.zaidi@hcuge.ch

Published online Jan. 25, 2018.

COPYRIGHT (C 2018 by the Society of Nuclear Medicine and Molecular Imaging. are unlikely to be delivered by medical imaging, the potential hazardous effects of radiation on the fetus include death, intrauterine growth limitation, average intelligence quotient loss, mental retardation, organ malformation, and small head size (1). Although controversial $(2,3)$, the linear no-threshold model is still the basis for radioprotection regulations. This model postulates that stochastic effects such as cancer might also occur at smaller doses (4-8). Because of this regulatory framework, and because health effects cannot be formally ruled out, the fetal absorbed doses should be known as precisely as possible. Estimating the fetal dose is, however, a challenging task, because of the difficulties associated with direct measurement of energy deposition and the irregular shape of the fetal body. Various approaches have been adopted to estimate the fetal dose, including Monte Carlo simulations using computational anthropomorphic models (7,917) and experimental measurements using physical phantoms with embedded dosimeters (18-21). However, these approaches inherently bear several limitations, such as the difficulty of constructing patient-specific computational models and of matching anthropomorphic physical phantoms to the size and location of the fetus within the maternal body (22). Because of these limitations, significant over- or underestimations of the fetal absorbed dose are possible. Monte Carlo calculations are considered the gold standard for dose estimation in diagnostic imaging (23), and their use in a clinical setting within a framework of patient-specific estimation of fetal dose from diagnostic procedures is highly desirable.

In this work, we expand on our previous set of anthropomorphic phantoms and describe the methodology for constructing patientspecific computational models for a pregnant female with twins. This model is based on a standardized hybrid pregnant-femaleand-fetus phantom, derived from an actual 25 weeks' pregnant patient who underwent an ${ }^{18} \mathrm{~F}$-FDG PET/CT scan while expecting twins. The patient-specific maternal and fetal absorbed radiation doses from both ${ }^{18} \mathrm{~F}-\mathrm{FDG}$ and CT were calculated and compared with the results provided by the OLINDA/EXM software (24) and the ImPACT CT (Impact Performance Assessment of CT, Bence Jones Offices, St. George's Hospital, London) dosimetry calculator, respectively. The influence of fetal position on fetal absorbed dose was also investigated.

\section{MATERIALS AND METHODS}

\section{Standardized Computational Model for Twins}

Our standardized pregnant female model with twins at the 25th wk of gestation was based on the International Commission on Radiological 


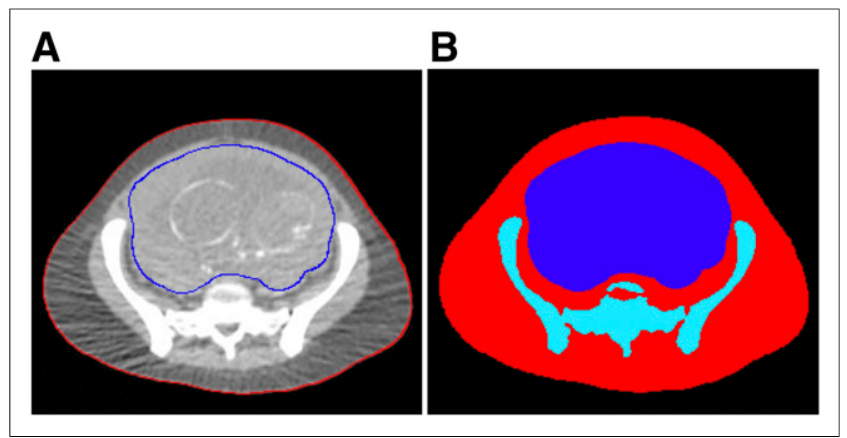

FIGURE 1. (A) CT image with external body contour (red line) around perimeter of pregnant patient and uterus (blue line) containing twin fetuses at 25th wk of gestation. (B) Segmented CT image with external body contour, skeleton, and uterus.

Protection (ICRP) computational pregnant female phantom at the 25th wk of gestation (17), which was developed using the mother torso and internal organs of the Rensselaer Polytechnic Institute phantoms (RPI-P) (25), the fetal and mother numeric models of Telecom ParisTech (26), the Katja model (27), and the newborn model of Helmholtz Zentrum München (28). The 25th-wk twins phantom was based on the fetal and mother numeric models and the Katja model. The organs of the fetus were scaled from the Katja model to match the reference organ masses of the ICRP phantom (29). The maternal bladder, small intestine, and large intestine were manually adjusted using the Rhinoceros package (Robert McNeel and Associates). The model includes 35 maternal tissues (adrenals, urinary bladder wall, urinary bladder content, brain, breasts, esophagus, eyeballs, eye lenses, gallbladder wall, gallbladder content, heart wall, heart content, kidneys, large intestine wall, large intestine content, liver, lungs, ovaries, pancreas, small intestine, skeleton, skin, spleen, stomach wall, stomach content, thymus, thyroid, trachea, uterine wall, uterine content, placenta, umbilical cord, amniotic fluid, yolk sac, and remainder tissues) and 50 fetal regions ( 25 for each fetus). The fetal tissue compositions were obtained from ICRP publication 89 (29): bone density was set at $1.3 \mathrm{~g} / \mathrm{cm}^{3}$ and soft-tissue density at $0.99 \mathrm{~g} / \mathrm{cm}^{3}$.

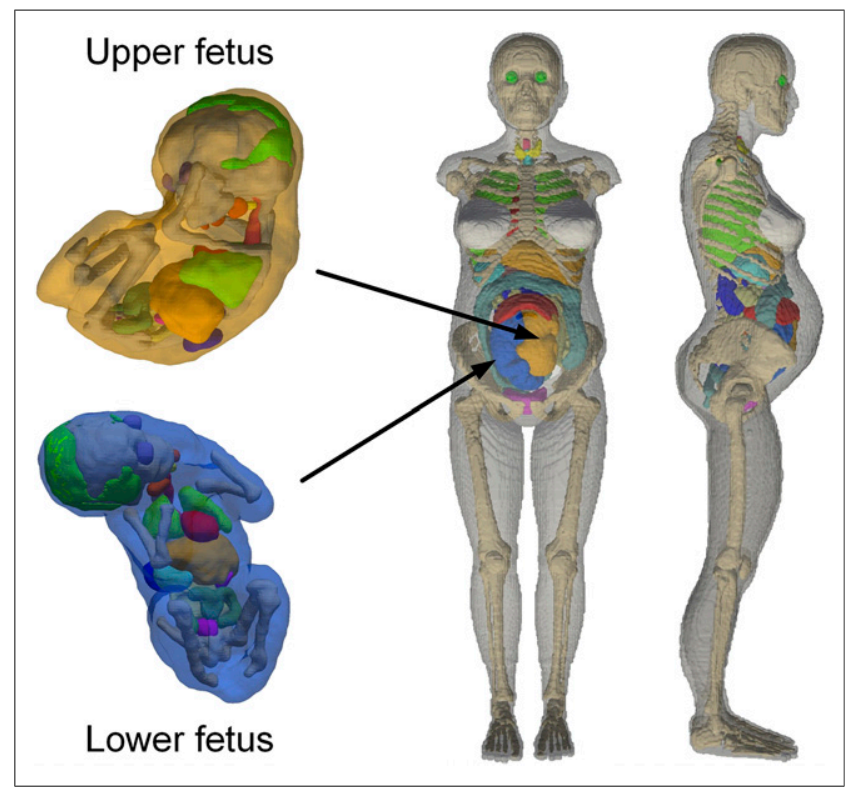

FIGURE 2. 3-dimensional views of computational pregnant female phantom at 25 weeks' gestation, with zoomed views of embedded twins.

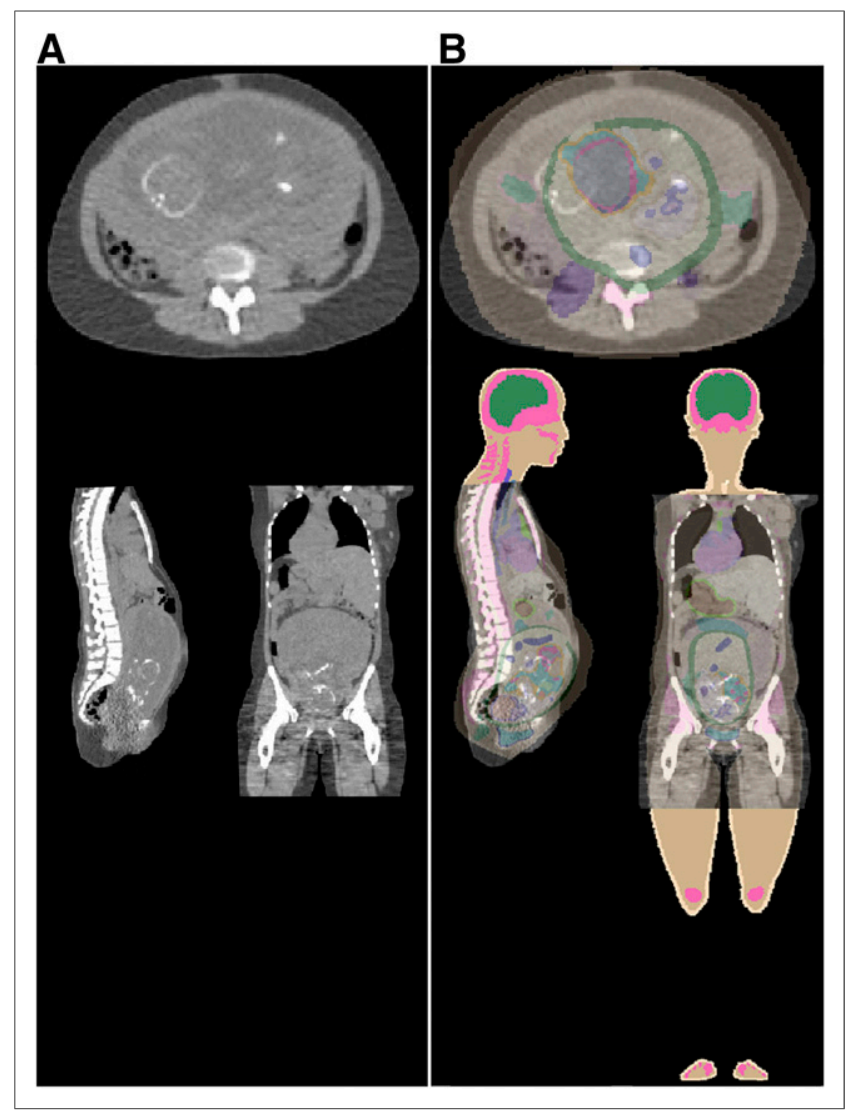

FIGURE 3. Representative slices showing image registration between abdominal CT images of patient (A) and computational phantom (B) for development of patient-specific pregnant computational models.

\section{Patient-Specific Computational Model}

The CT images of the pregnant patient were segmented into body, lung, skeleton, and uterus for constructing the regional voxel matrix (Fig. 1). This retrospective study, which was reported in previous work (30), did not require additional institutional review board approval, and the requirement to obtain informed consent was waived. First, the standardized twins computational model was voxelated from the boundary representation model. Then, using automatic affine registration via the Insight Toolkit (31), it was registered to the constructed

TABLE 1

Total Number of Disintegrations of ${ }^{18} \mathrm{~F}-\mathrm{FDG}$ in Maternal Organs and Fetuses

\begin{tabular}{ll}
\hline \multicolumn{1}{c}{ Organ } & $\begin{array}{c}\text { Total number of } \\
\text { disintegrations }(\mathrm{Bq} \mathrm{h} / \mathrm{Bq})\end{array}$ \\
\hline Brain & 0.21 \\
\hline Heart wall & 0.11 \\
\hline Lungs & 0.079 \\
\hline Liver & 0.13 \\
\hline Bladder & 0.3 \\
\hline Rest of maternal body & 1.7 \\
\hline Upper fetus & 0.0412 \\
\hline Lower fetus & 0.0434 \\
\hline
\end{tabular}


TABLE 2

Organ Masses of Fetuses

\begin{tabular}{|c|c|c|}
\hline \multirow[b]{2}{*}{ Organ } & \multicolumn{2}{|c|}{ Organ mass (g) } \\
\hline & Upper fetus & Lower fetus \\
\hline Soft tissue & 326.3 & 425.1 \\
\hline Skeleton & 117.3 & 148.4 \\
\hline Bone marrow & 8.8 & 13.5 \\
\hline Brain & 105.1 & 103.3 \\
\hline Esophagus & 3.5 & 2.8 \\
\hline Eyes & 1.9 & 2.7 \\
\hline Thyroid & 0.3 & 0.4 \\
\hline Spinal cord & 2.3 & 2.8 \\
\hline Lungs & 24.8 & 31.0 \\
\hline Heart & 5.4 & 8.3 \\
\hline Kidneys & 6.0 & 9.0 \\
\hline Liver & 33.0 & 44.3 \\
\hline Stomach & 9.5 & 11.3 \\
\hline Gallbladder & 0.7 & 1.5 \\
\hline Salivary gland & 1.5 & 3.4 \\
\hline Large intestine & 5.2 & 7.3 \\
\hline Small intestine & 8.9 & 13.0 \\
\hline Urinary bladder & 0.8 & 2.0 \\
\hline Skin & 104.9 & 147.4 \\
\hline Adrenal & 1.3 & 2.9 \\
\hline Pancreas & 2.7 & 4.5 \\
\hline Spleen & 1.2 & 1.6 \\
\hline Thymus & 2.8 & 5.1 \\
\hline Total body & 774.3 & 991.7 \\
\hline
\end{tabular}

CT-derived regional patient-specific voxel matrix to produce a new personalized computational model with well-defined anatomic structures that match patient CT images. The developed patient-specific computational model was used as input for Monte Carlo calculations of the radiation dose to the fetuses and maternal body. Figure 2 shows the 3-dimensional visualization of the constructed patient-specific model with zoomed views of the upper fetus and lower fetus. Figure 3 shows the registration of representative CT images between the patient and the standardized computational phantom for construction of the patient-specific model.

\section{Monte Carlo Simulations}

PET Component. The patient-specific computational phantom was imported to the Monte Carlo N-Particle Extended code (32) for radiation transport simulations. $\mathrm{S}$ values of uniformly distributed sources of ${ }^{18} \mathrm{~F}$ in maternal and fetal tissues were estimated. Calculation of the absorbed dose and effective dose delivered to both fetuses and the maternal organs from ${ }^{18} \mathrm{~F}-\mathrm{FDG}$ was based on the MIRD formalism (33). The regions of interest used to calculate the activity concentrations in the fetuses were manually drawn on the bodies of the fetuses (30). The time-integrated activity coefficients for ${ }^{18} \mathrm{~F}$-FDG in the maternal organs were obtained from ICRP publication 106 (34). Table 1 lists the total number of disintegrations (residence times) of ${ }^{18} \mathrm{~F}-\mathrm{FDG}$ in the maternal organs and the 2 fetuses. It was assumed that the average activity concentration in fetal tissues was the same in both fetuses. The mean total number of disintegrations of ${ }^{18} \mathrm{~F}-\mathrm{FDG}$ in the upper fetus and lower fetus were 0.0412 and $0.0434 \mathrm{~Bq} \mathrm{~h} / \mathrm{Bq}$, respectively.

CT Component. A GE Healthcare 750HD CT scanner model was created and validated using Monte Carlo-based techniques in our previous work $(12,35)$. This CT source and geometry model is equipped with a Performix Pro VCT $100 \mathrm{x}$-ray tube with $56^{\circ}$ fan-beam angle, a $7^{\circ}$ target angle, and a measured half-value layer of $7.8 \mathrm{~mm}$ of aluminum at $120 \mathrm{kVp}$. The patient-specific computational model was integrated with the CT scanner model in the N-Particle Extended code (32). Simulated CT examinations were performed using the personalized model and the protocol parameters used in our institution for patient scanning with a helical source path and a total collimation width of $64 \times 0.625 \mathrm{~mm}$ (12). The absorbed doses to maternal organs and fetal organs were calculated and normalized to the CT dose index $\left(\mathrm{CTDI}_{\mathrm{vol}}=1.8 \mathrm{mGy}\right)$ of the simulated examination (12).

\section{Absorbed Dose Calculations}

For the PET component, the absorbed radiation dose from ${ }^{18} \mathrm{~F}-\mathrm{FDG}$ to the target tissue $r_{T}$ is given by

$$
\mathrm{D}\left(r_{T}, T_{D}\right)=\sum_{r_{S}} A\left(r_{T}, T_{D}\right) S\left(r_{T} \leftarrow r_{S}\right)
$$

where $r_{S}$ is the source organ, $A\left(r_{T}, T_{D}\right)$ is the cumulated activity in the source organ over the dose-integration period $T_{D}$, and $S\left(r_{T} \leftarrow r_{S}\right)$ is the $S$ value describing the equivalent dose rate in the target organ per unit activity in the source organ.

For the CT component, the $\mathrm{CTDI}_{\mathrm{vol}}$-normalized absorbed radiation dose from the simulated examination to the clinical examination is estimated using the following equation:

$$
\frac{D_{r_{T}}^{\text {patient }}}{\mathrm{CTDI}_{\mathrm{vol}}^{\mathrm{patient}}}=\frac{D_{r_{T}}^{\mathrm{sim}}}{\mathrm{CTDI}_{\mathrm{vol}}^{\mathrm{sim}}},
$$

where $\mathrm{CTDI}_{\mathrm{vol}}^{\mathrm{sim}}$ is the $\mathrm{CTDI}_{\mathrm{vol}}$ of the patient in the simulated CT scanner, $\mathrm{D}_{r_{T}}^{\text {sim }}$ is the calculated absorbed dose to the patient in the simulated CT scanner, and $D_{r_{T}}^{\text {patient }} \mathrm{CTDI}_{\mathrm{vol}}^{\text {patient }}$ are the absorbed dose and $\mathrm{CTDI}_{\mathrm{vol}}$ to the patient in the clinical CT examinations, respectively. The reported $\mathrm{CTDI}_{\mathrm{vol}}$ for the patient in the clinical CT examination was $1.04 \mathrm{mGy}$. For the same patient model, the coefficient of

TABLE 3

\begin{tabular}{|c|c|c|c|c|c|}
\hline \multirow[b]{2}{*}{ Fetus } & \multicolumn{3}{|c|}{ Absorbed dose (mGy/MBq) from ${ }^{18} \mathrm{~F}-\mathrm{FDG}$} & \multicolumn{2}{|c|}{ Relative difference } \\
\hline & OLINDA/EXM 1.1 & OLINDA/EXM 2.0 & This work & $\begin{array}{l}\text { OLINDA/EXM } 1.1 \\
\text { vs. this work }\end{array}$ & $\begin{array}{l}\text { OLINDA/EXM } 2.0 \\
\text { vs. this work }\end{array}$ \\
\hline Upper & 0.00914 & 0.0121 & 0.0201 & $-54.50 \%$ & $-39.76 \%$ \\
\hline Lower & 0.00946 & 0.0125 & 0.0212 & $-55.45 \%$ & $-41.13 \%$ \\
\hline
\end{tabular}

Total-Body Doses from ${ }^{18} \mathrm{~F}$-FDG to Fetuses Compared with Those Obtained Using OLINDA/EXM 1.1 and OLINDA/EXM 2.0 


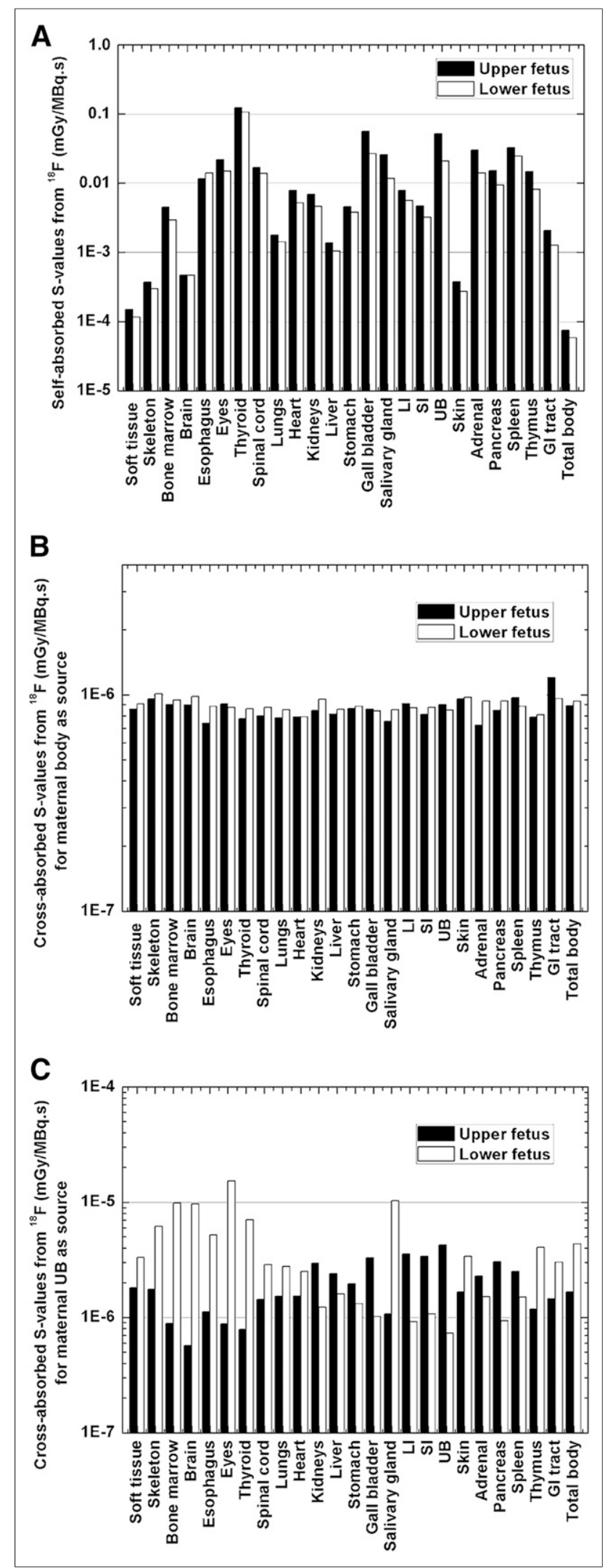

FIGURE 4. Self-absorbed $S$ values of ${ }^{18} \mathrm{~F}$ for fetal organs $(A)$ and cross-absorbed $S$ values of ${ }^{18} \mathrm{~F}$ from maternal body $(B)$ and maternal urinary bladder $(\mathrm{C})$, irradiating fetal bodies. $\mathrm{Gl}=$ gastrointestinal; $\mathrm{LI}=$ large intestine; $\mathrm{SI}=$ small intestine; $\mathrm{UB}=$ urinary bladder. variation for the $\mathrm{CTDI}_{\mathrm{vol}}$-normalized dose values across different scanners and protocols has been reported to be less than $10 \%(36)$.

\section{RESULTS}

\section{Patient-Specific Computational Phantom}

The accuracy of the registration between the regional voxel matrix of the patient and the generated patient-specific computational phantom was evaluated by calculating the Jaccard coefficient of similarity (37) for the corresponding segmented organs: $0.73,0.60,0.12$ and 0.55 for total body, lung, skeleton and uterus, respectively. The low Jaccard coefficient for the skeleton was caused mainly by the mismatch of the ribs and hip bones between the anchor phantom and patient. The weight of the lower fetus matched well the ICRP-recommended fetal weight of $990 \mathrm{~g}$ at 25 weeks' gestation, and the weight of the upper fetus was slightly lower (Table 2).

\section{Absorbed Doses from the PET Component}

The coefficient of variation for ${ }^{18} \mathrm{~F}$-FDG organ dose between the 2 fetuses was approximately $8.5 \%$, with a minimum of approximately $0.3 \%$ (for the spinal cord) and a maximum of approximately $23.2 \%$ (for the eyes), whereas the mean differences in the fetal total-body dose between this work and the 2 versions of OLINDA/EXM were $-55 \%$ and $-40.5 \%$, respectively (Table 3 ). As shown in Figure 4A, for the same organ, the estimated selfabsorbed $\mathrm{S}$ value of the lower fetus was about $29 \%$ smaller than the value of the upper fetus. Indeed, the self-absorbed $S$ values of fetal organs decrease when fetal weight increases (17). The crossabsorbed $S$ values were obtained assuming uniform activity in all maternal source tissues. As shown in Figures 4B and 4C, the crossabsorbed $\mathrm{S}$ values for the maternal body irradiating the fetus were contributed mostly by the annihilation photons and presented a uniform distribution in different fetal organs. The mean absolute difference in cross-absorbed $\mathrm{S}$ values from the maternal body between the 2 fetuses was about $8 \%$. The cross-absorbed $\mathrm{S}$ values from maternal urinary bladder were affected by the fetal position

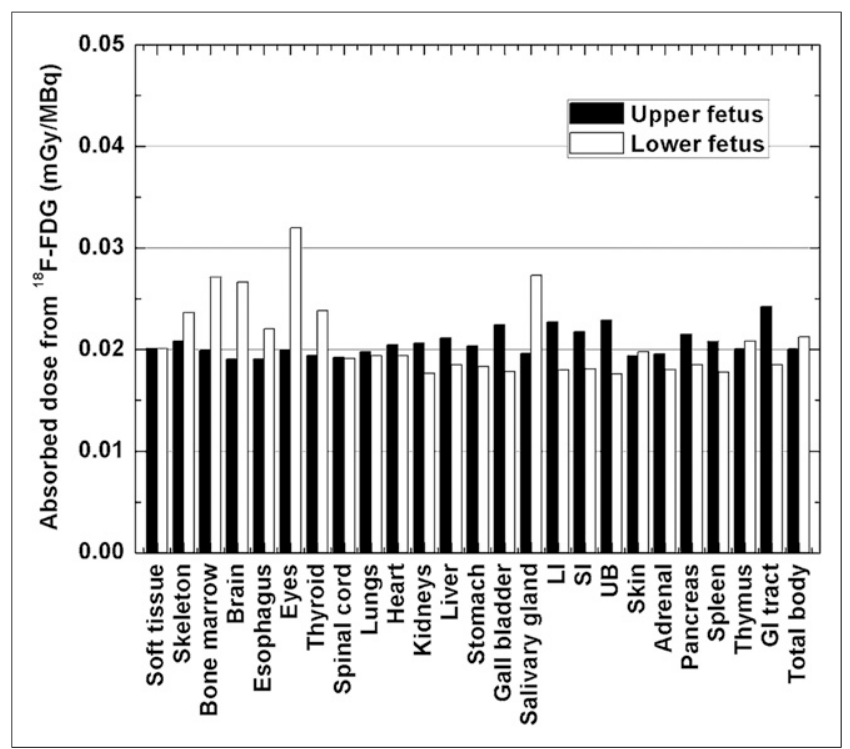

FIGURE 5. Absorbed doses to different organs of fetuses from ${ }^{18} \mathrm{~F}-$ FDG. GI = gastrointestinal; $\mathrm{LI}=$ large intestine; $\mathrm{SI}=$ small intestine; $\mathrm{UB}=$ urinary bladder. 


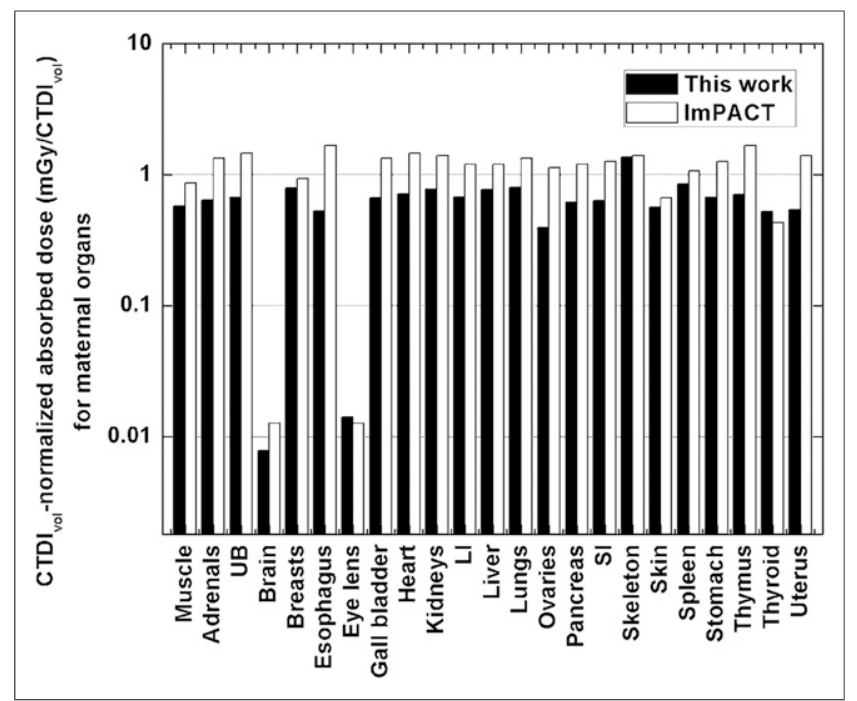

FIGURE 6. Normalized absorbed doses to different organs of pregnant patient from CT examination. $\mathrm{LI}=$ large intestine; $\mathrm{SI}=$ small intestine; UB = urinary bladder.

and presented a mean absolute difference of $112 \%$ between the 2 fetuses. Figure 5 illustrates the organ-absorbed doses in the 2 fetuses from ${ }^{18} \mathrm{~F}-\mathrm{FDG}$. In the upper fetus the gastrointestinal tract received the highest absorbed dose, whereas in the lower fetus the absorbed dose to the eyes and bone marrow was higher than in the other organs. The absorbed ${ }^{18} \mathrm{~F}$-FDG doses to the upper and lower fetuses were 0.0201 and $0.0212 \mathrm{mGy} / \mathrm{MBq}$, respectively. The absorbed dose to the maternal uterus was $11.4 \%$ and $16.2 \%$ lower than the total-body dose to the upper and the lower fetuses, respectively.

\section{Absorbed Doses from the CT Component}

Except for the total body, skeleton, and bone marrow, the absorbed CT doses to other fetal organs were about $20 \%$ lower than the dose to the maternal uterus. The variation in organ CT dose between the 2 fetuses was approximately 5.7\%, with a minimum of approximately $0.3 \%$ (for the liver) and a maximum

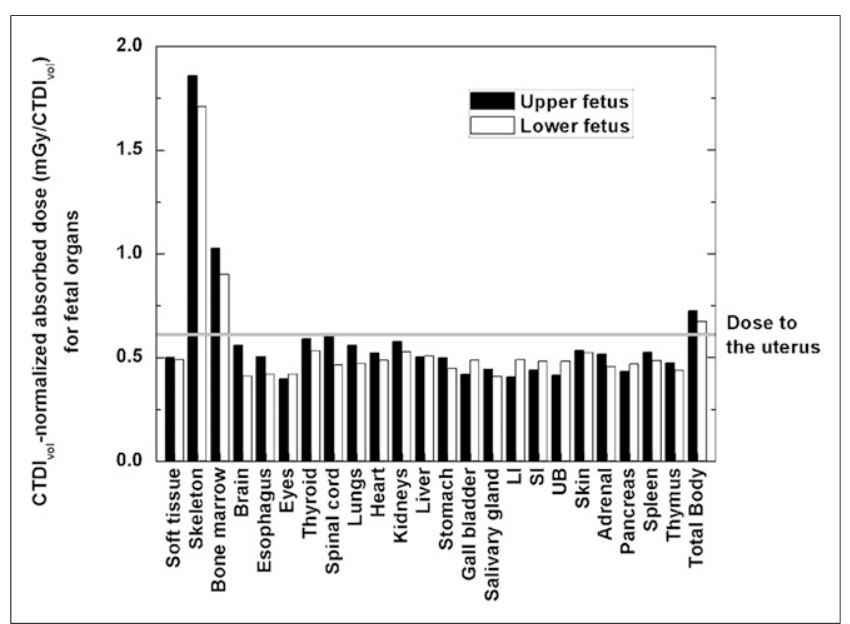

FIGURE 7. Normalized absorbed doses to different organs of fetuses from $\mathrm{CT}$ examination. $\mathrm{LI}=$ large intestine; $\mathrm{SI}=$ small intestine; $\mathrm{UB}=$ urinary bladder.
TABLE 4

Absorbed Dose and Effective Dose to Maternal Body from PET/CT Examination

\begin{tabular}{lccc}
\hline & \multicolumn{3}{c}{ Absorbed dose (mGy) } \\
\cline { 2 - 4 } \multicolumn{1}{c}{ Maternal organ } & CT & PET & \\
\hline Remainder & 0.60 & 2.31 & 2.91 \\
\hline Adrenals & 0.67 & 2.96 & 3.62 \\
\hline Urinary bladder & 0.70 & 13.02 & 13.72 \\
\hline Brain & 0.01 & 7.77 & 7.78 \\
\hline Breasts & 0.82 & 2.01 & 2.84 \\
\hline Esophagus & 0.55 & 3.06 & 3.61 \\
\hline Eye lens & 0.01 & 2.13 & 2.14 \\
\hline Gallbladder & 0.69 & 3.34 & 4.03 \\
\hline Heart wall & 0.74 & 16.35 & 17.08 \\
\hline Kidneys & 0.81 & 2.76 & 3.57 \\
\hline Large intestine wall & 0.71 & 2.88 & 3.59 \\
\hline Liver & 0.80 & 5.22 & 6.02 \\
\hline Lungs & 0.84 & 3.93 & 4.76 \\
\hline Ovaries & 0.41 & 3.22 & 3.63 \\
\hline Pancreas & 0.64 & 2.99 & 3.63 \\
\hline Small intestine & 0.66 & 2.86 & 3.52 \\
\hline Skeleton & 1.41 & 2.48 & 3.89 \\
\hline Skin & 0.59 & 1.70 & 2.28 \\
\hline Spleen & 0.88 & 2.65 & 3.53 \\
\hline Stomach & 0.70 & 3.14 & 3.85 \\
\hline Thymus & 0.73 & 3.97 & 4.71 \\
\hline Thyroid & 0.55 & 2.20 & 2.75 \\
\hline Uterus & 0.57 & 3.35 & 3.91 \\
\hline Total body & 0.72 & 2.83 & 3.55 \\
\hline Effective dose (mSv) & 0.79 & 3.62 & 4.41 \\
\hline & & & \\
\hline & & & \\
\hline & & & \\
\hline
\end{tabular}

of approximately $15.2 \%$ (for the brain). Figure 6 compares the $\mathrm{CTDI}_{\mathrm{vol}}$-normalized absorbed dose for different maternal organs with estimates provided by the ImPACT CT dosimetry calculator. The mean absolute difference in dose to the maternal organs between this work and ImPACT CT was $40.3 \%$. Figure 7 shows the $\mathrm{CTDI}_{\mathrm{vol}}$-normalized absorbed dose from the CT component to fetal organs. The absorbed dose was nonuniformly distributed between fetal organs. For instance, the absorbed dose to the fetal skeleton and bone marrow was about 3.7 and 2.0 times higher, respectively, than that received by other fetal organs. Because a lowdose CT protocol was performed, the absorbed doses to the 2 fetuses were relatively low, at 0.73 and $0.67 \mathrm{mGy} / \mathrm{CTDI}_{\mathrm{vol}}$, respectively, whereas the absorbed dose to the uterus was $0.61 \mathrm{mGy} / \mathrm{CTDI}_{\mathrm{vol}}$.

\section{Total Absorbed Doses from the Combined PET/ CT Examination}

The absorbed dose to the upper fetus was $0.76 \mathrm{mGy}$ from the CT component and $3.78 \mathrm{mGy}$ from the PET component, for a total of $4.53 \mathrm{mGy}$ from the PET/CT examination, whereas the absorbed dose to the lower fetus was $0.70 \mathrm{mGy}$ from the CT component and $3.99 \mathrm{mGy}$ from the PET component, for a total of $4.69 \mathrm{mGy}$ from the PET/CT examination. For the different fetal organs, the CT 
TABLE 5

Absorbed Dose to Fetuses from PET/CT Examination

\begin{tabular}{|c|c|c|c|c|c|c|}
\hline \multirow[b]{2}{*}{ Fetal organ } & \multicolumn{2}{|c|}{ CT component } & \multicolumn{2}{|c|}{ PET component } & \multicolumn{2}{|c|}{ Total } \\
\hline & Upper fetus & Lower fetus & Upper fetus & Lower fetus & Upper fetus & Lower fetus \\
\hline Soft tissue & 0.52 & 0.51 & 3.79 & 3.78 & 4.31 & 4.29 \\
\hline Skeleton & 1.94 & 1.78 & 3.92 & 4.45 & 5.86 & 6.23 \\
\hline Bone marrow & 1.07 & 0.94 & 3.75 & 5.10 & 4.81 & 6.04 \\
\hline Brain & 0.58 & 0.43 & 3.59 & 5.01 & 4.17 & 5.44 \\
\hline Esophagus & 0.53 & 0.44 & 3.58 & 4.14 & 4.11 & 4.58 \\
\hline Eyes & 0.41 & 0.44 & 3.75 & 6.02 & 4.17 & 6.46 \\
\hline Thyroid & 0.62 & 0.55 & 3.66 & 4.48 & 4.27 & 5.03 \\
\hline Spinal cord & 0.64 & 0.48 & 3.62 & 3.59 & 4.26 & 4.08 \\
\hline Lungs & 0.58 & 0.49 & 3.72 & 3.64 & 4.30 & 4.14 \\
\hline Heart & 0.54 & 0.51 & 3.85 & 3.64 & 4.39 & 4.15 \\
\hline Kidneys & 0.60 & 0.55 & 3.88 & 3.32 & 4.48 & 3.87 \\
\hline Liver & 0.53 & 0.53 & 3.98 & 3.48 & 4.51 & 4.01 \\
\hline Stomach & 0.52 & 0.47 & 3.83 & 3.45 & 4.35 & 3.92 \\
\hline Gallbladder & 0.44 & 0.51 & 4.22 & 3.35 & 4.66 & 3.86 \\
\hline Salivary gland & 0.46 & 0.43 & 3.69 & 5.14 & 4.15 & 5.56 \\
\hline Large intestine & 0.42 & 0.51 & 4.27 & 3.39 & 4.70 & 3.90 \\
\hline Small intestine & 0.46 & 0.50 & 4.09 & 3.40 & 4.55 & 3.90 \\
\hline Urinary bladder & 0.43 & 0.50 & 4.30 & 3.31 & 4.73 & 3.81 \\
\hline Skin & 0.56 & 0.54 & 3.65 & 3.72 & 4.21 & 4.27 \\
\hline Adrenal & 0.54 & 0.48 & 3.68 & 3.39 & 4.22 & 3.87 \\
\hline Pancreas & 0.45 & 0.49 & 4.05 & 3.48 & 4.50 & 3.97 \\
\hline Spleen & 0.55 & 0.51 & 3.91 & 3.34 & 4.46 & 3.85 \\
\hline Thymus & 0.49 & 0.46 & 3.77 & 3.92 & 4.27 & 4.37 \\
\hline Total body & 0.76 & 0.70 & 3.78 & 3.99 & 4.53 & 4.69 \\
\hline
\end{tabular}

component contributed $8.4 \%-30.8 \%$ of the total absorbed dose whereas the PET component contributed $70 \%-91.6 \%$ of the total absorbed dose. For the patient, the injected ${ }^{18} \mathrm{~F}-\mathrm{FDG}$ activity was $188 \mathrm{MBq}$, and the patient-specific $\mathrm{CTDI}_{\mathrm{vol}}$ performed in the CT examination was $1.04 \mathrm{mGy}$ without tube current modulation. The calculated absolute doses to the patient and fetuses are summarized in Tables 4 and 5, respectively. The patient's effective dose was $0.79 \mathrm{mSv}$ from the CT component and $3.62 \mathrm{mSv}$ from the PET component, resulting in a total of $4.41 \mathrm{mSv}$ from the PET/CT examination.

\section{DISCUSSION}

We have described our methodologic procedure to create a computational patient-specific phantom enabling calculation of the fetal dose in the particular case of twin pregnancy. Our approach, which involves coregistration of clinical images to the computational phantom followed by Monte Carlo-based dose calculations, is suitable for individualized dose assessment in a clinical setting. The unusual anatomy of a twin pregnancy is a clear example of the adaptability of our methodology to disparate clinical situations.

In calculation of the fetal dose, there were 3 main sources of inaccuracy: from the geometric point of view, the registration of the standardized phantom to the clinical images; the uncertainty linked to Monte Carlo simulations; and the $\mathrm{CTDI}_{\mathrm{vol}}$-based estimation of the dose to the organs. The uncertainty from the image registration process was by far the greatest source of inaccuracy, accounting for $27 \%-45 \%$ of the variability in the dose, whereas the error expected from Monte Carlo calculations and $\mathrm{CTDI}_{\mathrm{vol}}$-based organ dose estimation was generally less than $5 \%$. The $\mathrm{CTDI}_{\mathrm{vol}}$ and the dose-length product are the 2 metrics commonly conveyed in dose reports by commercial CT scanners and used to determine CT doses to pregnant patients and fetus (12).

The estimation of the CT dose showed that the fetal skeleton and bone marrow receive a substantially higher dose than soft tissues because of the higher density of the former. Although never proven for the range of doses received during medical examinations, radiation exposure of the bone marrow in childhood creates the hypothetical risk that malignancies will develop later in life, and this aspect should be considered when the acquisition protocol is designed for the CT component.

We investigated the influence of anatomic characteristics of the fetus on radiation dosimetry by comparing the organ dose of the 2 fetuses. For the PET component, the coefficient of variation across the 2 fetuses for a given organ ranged from $0.3 \%$ (for the spinal cord) to $23.2 \%$ (for the eyes), with a mean across all organs of $8.5 \%$. For the CT component, the coefficient of variation across the 2 fetuses for a given organ ranged from $0.3 \%$ (for the liver) to $15.2 \%$ (for the brain), with a mean across all organs of $5.7 \%$. These results indicate that, at the same stage of gestation, 
anatomic differences between the fetuses influence the estimation of the internal and external absorbed doses for any organ. The organ dose to a particular fetus would be within approximately $25 \%$ of the mean value across the whole fetus at the same stage of gestation. From a radiobiologic point of view, this difference in absorbed dose between the 2 fetuses is likely to be insignificant.

An important finding of our study was that the fetal doses estimated using either OLINDA/EXM 1.1 or 2.0 were about $50 \%$ smaller than the doses calculated using our patient-specific model. This discrepancy can be attributed mainly to the differences between the fetal weights and source-to-fetus distances used in our personalized computational phantom and patient images and those used in OLINDA/EXM 1.1 and 2.0, which are based on a stylized model (38) and the 6-mo voxel-based RPI-P model (25), respectively. When generic anthropomorphic phantoms, such as those included in both versions of OLINDA/EXM, are used, the final dose is heavily dependent on the characteristics of the phantom, such as the mass and the geometric arrangement of the organs. The use of a patient-specific phantom in a clinical setting allows more accurate dose calculation, which is of particular importance in cases such as pregnant women or pretherapeutic imaging. In radiation dosimetry, the absorbed dose to the maternal uterus is used as a conservative proxy for the fetal dose (39). This work showed that the mean fetal dose was about $15 \%$ higher than the uterine dose for both the PET and the CT components. For CT, the uterine dose may still be used as a conservative value for fetal soft tissues (i.e., except for the skeleton and bone marrow).

Our study showed that the ${ }^{18} \mathrm{~F}-\mathrm{FDG}$ contribution to the total dose was much higher than the CT contribution, for both the mother and the fetuses (i.e., about $16 \%-18 \%$ vs. $82 \%-84 \%$ ). In addition, the maximum contribution to the organ dose from CT was about $30 \%$ to the fetal skeleton, whereas the maximum contribution from ${ }^{18}$ F-FDG was more than $90 \%$ to the brain, eyes, and salivary gland. In total, the mean fetal dose from the PET/CT examination was $4.61 \mathrm{mGy}$. This level of radiation not only is well below the threshold of deterministic effects but also is below the threshold where stochastic effects have been documented in humans (40). In imaging of pregnant women, priority should be given to achieving an examination of diagnostic quality, because overly aggressive dose-reduction protocols may put the life of both the mother and the fetus in danger (41).

\section{CONCLUSION}

The proposed approach for constructing personalized computational models enables calculation of a patient-specific radiation dose during a combined PET/CT examination, even in complex, unusual situations such as a patient pregnant with twins. The fetal organ dose is particularly affected by the fetal position, among other factors. The calculated personalized ${ }^{18}$ F-FDG fetal dose was significantly higher than the corresponding CT dose and was about $40 \%-50 \%$ higher than doses reported by widely used popular internal dosimetry software.

\section{DISCLOSURE}

This work was supported by the Swiss National Science Foundation under grant SNSF 320030_176052. No other potential conflict of interest relevant to this article was reported.

\section{ACKNOWLEDGMENTS}

We thank Drs X. George Xu (Rensselaer Polytechnic Institute), Isabelle Bloch (Telecom ParisTech), and Maria Zankl (Helmholtz Zentrum München) for providing the computational models.

\section{REFERENCES}

1. McCollough $\mathrm{CH}$, Schueler BA, Atwell TD, et al. Radiation exposure and pregnancy: when should we be concerned? Radiographics. 2007;27:909917.

2. Siegel JA, Sacks B. Eliminating use of the linear no-threshold assumption in medical imaging. J Nucl Med. 2017;58:1014-1015.

3. Tirada N, Dreizin D, Khati NJ, Akin EA, Zeman RK. Imaging pregnant and lactating patients. Radiographics. 2015;35:1751-1765.

4. ICRP. Publication 84: pregnancy and medical radiation. Ann ICRP. 2000;30: iii-viii, $1-43$.

5. ICRP. Publication 90: biological effects after prenatal irradiation (embryo and fetus). Ann ICRP. 2003;33:5-206.

6. Pearce MS, Salotti JA, Little MP, et al. Radiation exposure from CT scans in childhood and subsequent risk of leukaemia and brain tumours: a retrospective cohort study. Lancet. 2012;380:499-505.

7. Helmrot E, Pettersson H, Sandborg M, Alten JN. Estimation of dose to the unborn child at diagnostic $\mathrm{x}$-ray examinations based on data registered in RIS/ PACS. Eur Radiol. 2007;17:205-209.

8. ACR-SPR Practice Parameter for Imaging Pregnant or Potentially Pregnant Adolescents and Women with Ionizing Radiation. Revised 2013 (resolution 48). Reston, VA: American College of Radiology; 2013.

9. Angel E, Wellnitz CV, Goodsitt MM, et al. Radiation dose to the fetus for pregnant patients undergoing multidetector CT imaging: Monte Carlo simulations estimating fetal dose for a range of gestational age and patient size. Radiology. 2008;249:220-227.

10. Damilakis J, Perisinakis K, Tzedakis A, Papadakis AE, Karantanas A. Radiation dose to the conceptus from multidetector CT during early gestation: a method that allows for variations in maternal body size and conceptus position. Radiology. 2010;257:483-489.

11. Damilakis J, Tzedakis A, Perisinakis K, Papadakis AE. A method of estimating conceptus doses resulting from multidetector CT examinations during all stages of gestation. Med Phys. 2010;37:6411-6420.

12. Xie T, Poletti PA, Platon A, Becker CD, Zaidi H. Assessment of CT dose to the fetus and pregnant female patient using patient-specific computational models. Eur Radiol. 2018;28:1054-1065.

13. Gu J, Xu XG, Caracappa PF, Liu B. Fetal doses to pregnant patients from CT with tube current modulation calculated using Monte Carlo simulations and realistic phantoms. Radiat Prot Dosimetry. 2013;155:64-72.

14. Lopez-Rendon X, Walgraeve MS, Woussen S, et al. Comparing different methods for estimating radiation dose to the conceptus. Eur Radiol. 2017;27:851858 .

15. Maynard MR, Long NS, Moawad NS, et al. The UF family of hybrid phantoms of the pregnant female for computational radiation dosimetry. Phys Med Biol. 2014;59:4325-4343.

16. Winer-Muram HT, Boone JM, Brown HL, Jennings SG, Mabie WC, Lombardo GT. Pulmonary embolism in pregnant patients: fetal radiation dose with helical CT. Radiology. 2002;224:487-492.

17. Xie T, Zaidi H. Development of computational pregnant female and fetus models and assessment of radiation dose from positron-emitting tracers. Eur J Nucl Med Mol Imaging. 2016;43:2290-2300.

18. Chatterson LC, Leswick DA, Fladeland DA, Hunt MM, Webster S, Lim H. Fetal shielding combined with state of the art CT dose reduction strategies during maternal chest CT. Eur J Radiol. 2014;83:1199-1204.

19. Jaffe TA, Neville AM, Anderson-Evans C, et al. Early first trimester fetal dose estimation method in a multivendor study of 16- and 64-MDCT scanners and low-dose imaging protocols. AJR. 2009;193:1019-1024.

20. Kelaranta A, Kaasalainen T, Seuri R, Toroi P, Kortesniemi M. Fetal radiation dose in computed tomography. Radiat Prot Dosimetry. 2015;165:226-230.

21. Solomou G, Papadakis AE, Damilakis J. Abdominal CT during pregnancy: a phantom study on the effect of patient centering on conceptus radiation dose and image quality. Eur Radiol. 2015;25:911-921.

22. Zaidi H, Xu XG. Computational anthropomorphic models of the human anatomy: the path to realistic Monte Carlo modeling in medical imaging. Annu Rev Biomed Eng. 2007;9:471-500.

23. Zaidi H, Ay M. Current status and new horizons in Monte Carlo simulation of X-ray CT scanners. Med Biol Eng Comput. 2007;45:809-817. 
24. Stabin MG, Sparks RB, Crowe E. OLINDA/EXM: the second-generation personal computer software for internal dose assessment in nuclear medicine. $\mathrm{J} \mathrm{Nucl}$ Med. 2005;46:1023-1027.

25. Xu XG, Taranenko V, Zhang J, Shi C. A boundary-representation method for designing whole-body radiation dosimetry models: pregnant females at the ends of three gestational periods-RPI-P3, -P6 and -P9. Phys Med Biol. 2007;52: 7023-7044.

26. Bibin L, Anquez J, de la Plata Alcalde JP, Boubekeur T, Angelini ED, Bloch I. Whole-body pregnant woman modeling by digital geometry processing with detailed uterofetal unit based on medical images. IEEE Trans Biomed Eng. 2010; 57:2346-2358.

27. Becker J, Zankl M, Fill U, Hoeschen C. Katja: the 24th week of virtual pregnancy for dosimetric calculations. Pol J Med Phys Eng. 2008;14:13-20.

28. Petoussi-Henss N, Zankl M, Fill U, Regulla D. The GSF family of voxel phantoms. Phys Med Biol. 2002;47:89-106.

29. Publication 89: basic anatomical and physiological data for use in radiological protection: reference values. Ann ICRP. 2002;32:5-265.

30. Zanotti-Fregonara P, Chastan M, Edet-Sanson A, et al. New fetal dose estimates from ${ }^{18} \mathrm{~F}$-FDG administered during pregnancy: standardization of dose calculations and estimations with voxel-based anthropomorphic phantoms. J Nucl Med. 2016;57:1760-1763.

31. Johnson HJ, McCormick MM, Ibáñez L, and the Insight Software Consortium. The ITK Software Guide. 2nd ed. Clifton Park, NY: Kitware Inc.; 2005.

32. Pelowitz DB. MCNPX User's Manual. Version 2.5.0. Los Alamos, NM: Los Alamos National Laboratory; 2005. LA-CP-05-0369.
33. Bolch WE, Eckerman KF, Sgouros G, Thomas SR. MIRD pamphlet no. 21: a generalized schema for radiopharmaceutical dosimetry-standardization of nomenclature. J Nucl Med. 2009;50:477-484.

34. Publication 106: radiation dose to patients from radiopharmaceuticals-addendum 3 to ICRP publication 53. Ann ICRP. 2008;38:1-197.

35. Akbarzadeh A, Ay MR, Ghadiri H, Sarkar S, Zaidi H. Measurement of scattered radiation in a volumetric 64-slice CT scanner using three experimental techniques. Phys Med Biol. 2010;55:2269-2280.

36. Turner AC, Zankl M, DeMarco JJ, et al. The feasibility of a scanner-independent technique to estimate organ dose from MDCT scans: using CTDIvol to account for differences between scanners. Med Phys. 2010;37:1816-1825.

37. Jackson DA, Somers KM, Harvey HH. Similarity coefficients: measures of cooccurrence and association or simply measures of occurrence? Am Nat. 1989;133: 436-453.

38. Stabin M, Watson E, Cristy M, et al. Mathematical Models and Specific Absorbed Fractions of Photon Energy in the Nonpregnant Adult Female and at the End of Each Trimester of Pregnancy. Oak Ridge, TN: Oak Ridge National Laboratory; 1995. Report ORNL/TM-12907.

39. Petoussi-Henss N, Schlattl H, Zankl M, Endo A, Saito K. Organ doses from environmental exposures calculated using voxel phantoms of adults and children. Phys Med Biol. 2012;57:5679-5713.

40. Brent RL. Protection of the gametes embryo/fetus from prenatal radiation exposure. Health Phys. 2015;108:242-274.

41. Zanotti-Fregonara P, Hindie E. Performing nuclear medicine examinations in pregnant women. Phys Med. 2017;43:159-164. 